\title{
Thermoset and Thermoplastic Elastomeric Chain Comparative Strength: An In Vitro Study
}

\author{
Ari Triwardhani, Vindira P. Prastiti, Iyanda Vemala, Jusuf Sjamsudin \\ Department of Orthodontics, Faculty of Dental Medicine, Universitas Airlangga, Surabaya, Indonesia
}

\section{Abstract}

\begin{abstract}
Aim: Technology in the field of orthodontic has experienced many developments in the last two decades. The ultimate goal of orthodontic treatment is to provide continuous light pressure to get orthodontic tooth movement with minimum side effects. Elastomeric chains are commonly used materials for moving teeth and closing space. The strength of elastomeric chain results in a tendency for better elastomer selection. Some factories make elastomeric chains with thermoplastic and thermoset materials with thermoset material claims having strength resistance for longer periods of time. The aim of the study was to compare the degradation strength between thermoplastic and thermoset elastomeric chain. Materials and Methods: This was a true-experimental laboratory study (in vitro study). The sample comprised open-type elastomeric chains (power chain) with clear transparent color thermoplastic material, which include original power chain, plastic chain and thermoset material generation II, and memory chain. The samples were divided into four groups $(n=5): 24 \mathrm{~h}, 7,14$, and 21 days. Independent samples $t$ tests and analysis of variance (ANOVA) were performed to analyze the data. Results: There was significant dissimilarity between thermoplastic and thermoset elastomeric chain material in strength. Thermoplastic elastomeric chains decreased by $60.84 \%-65.51 \%$ and thermoset elastomeric chain reached $36.28 \%-58.80 \%$ within 21 days in artificial saliva solution. Conclusion: There was dissimilarity between the thermoplastic and thermoset elastomeric chain materials in force decay due to excessive early withdrawal forces in the elastomeric chains of thermoplastic materials with a more rigid material than the thermoset elastomeric chains of the thermoset material.
\end{abstract}

Keywords: Comparative Strength, Elastomeric Chains, Thermoplastic, Thermoset

Received: 13-02-2020, Revised: 16-04-2020, Accepted: 19-04-2020, Published: 21-10-2020.

\section{INTRODUCTION}

Technology in the field of orthodontics has experienced many developments in the last two decades including the process of making braces, brackets, bonding techniques, and elastomeric materials. This development has contributed to orthodontic treatment, which will facilitate the application of pressure strength, direction of tooth movement, and desired duration.$^{[1,2]}$ Elastomeric chains are a material commonly used to move teeth and close spaces. The advantages of elastomeric chains include easy to use, affordable prices, reduces the potential for intraoral trauma, and have a variety of colors or transparent. ${ }^{[1]}$

Previous experiments about elastomeric chain mechanical properties are seen from the decrease in strength over time and the level of activation, simulation of space closure,

\begin{tabular}{|l|l|}
\hline \multicolumn{2}{|c|}{ Access this article online } \\
\hline Quick Response Code: & Website: \\
& www.jioh.org \\
\cline { 2 - 2 } & \\
\hline
\end{tabular}

pre-stretching elastomeric chains, environmental factors, storage, and design of elastomeric chains. ${ }^{[3]}$ This difference can be influenced by a variety of manufacturing techniques including cutting or injection molding of raw materials, the effects of some additives incorporated in the final product, and differences in shape (ellipse or round) or dimensional characteristics (whether or not there are inter-modular links) of the elastomeric chains. ${ }^{[2,4]}$ Elastomeric chains are not ideal elastic because they can experience permanent deformation and decrease in strength over time. Deformation mechanisms are related to chain slippage and molecular stretching. ${ }^{[5]}$

Address for correspondence: Dr. Ari Triwardhani, Department of Orthodontics, Faculty of Dental Medicine Universitas Airlangga, Surabaya, Indonesia E-mail: ari-t@fkg.unair.ac.id

This is an open access journal, and articles are distributed under the terms of the Creative Commons Attribution-NonCommercial-ShareAlike 4.0 License, which allows others to remix, tweak, and build upon the work non-commercially, as long as appropriate credit is given and the new creations are licensed under the identical terms.

For reprints contact: reprints@medknow.com

How to cite this article: Triwardhani A, Prastiti VP, Vemala I, Sjamsudin J. Thermoset and thermoplastic elastomeric chain comparative strength: An in vitro study. J Int Oral Health 2020;12:485-90. 
Thermoplastic and thermoset chains are different manufacturer's materials of elastomeric chains. Thermoplastic is a plastic that is softened by heating and will harden when cooled. Thermoplastic is formed from linear molecular rings and is formed by mold without chemical processes before printing. The most important characteristic of thermoplastic is the glass temperature transition when softening. Thermoplastic polymers, such as polyethylene, are types of polymers that have thermoplastic properties caused by a linear, branched, or a cross-linked chain structure. Polymers of this type will be soft and viscous when heated and become hard and rigid when repeatedly cooled. ${ }^{[6]}$ Thermosets are polymers formed by chemical reactions or heat so that they cannot be liquefied and dissolved. Thermoset polymers have irreversible polymerization with cross-molecular structures and are formed in two stages of polymerization. The first step is the formation of polymers with linear rings. The second stage of polymerization produces a cross-linking structure. The end product can be made hard or flexible. ${ }^{[7]}$

The strength reduction obtained in several kinds of literature has different protocols taken from the evaluation of the degree of strength reduction including wet or dry tests, including water, artificial saliva, or fluoride media in acidic or neutral $\mathrm{pH}$, different temperatures, and application of reduced or stable pressure. The aim of this study is to compare the degradation strength between thermoplastic and thermoset elastomeric chain. ${ }^{[2]}$

\section{Materials and Methods \\ Setting and design}

True experimental study with posttest-only control group design and in vitro study were conducted to investigate the comparison of thermoset and thermoplastic elastomeric ligature strength. This research was conducted at Research Center Laboratory of Dental Medicine, Airlangga University for 1 month. The remaining forces and the dimensional changes were measured at different time points over a period of $24 \mathrm{~h}, 7,14$, and 21 days.

\section{Sample preparation}

The sample of this study used the clear and short elastomeric chain from American Orthodontics [AO], Sheboygan, Wisconsin, United States) and Research and Manufacturing Company (Ormco, Glendora, California, United States). Four elastomeric chains were selected for evaluation: (1) Ormco Colored Power Chains-thermoplastic (OR 1); (2) Ormco Generation II Power Chains-thermoset (OR 2); (3) AO Plastic Chainsthermoplastic (AO 1); and (4) AO T-Memory Chainsthermoset (AO 2). The sample of 20 thermoplastic and thermoset elastomeric chains was divided into four groups $(n=5)$ to record the force loss at four time points: $24 \mathrm{~h}, 7$, 14 , and 21 days.

Specimens were mounted on custom test jigs. Twenty jigs, each with a series of pins set $20 \mathrm{~mm}$ apart, were used to hold stretched elastomeric chains at a constant length [Figure 1]. These jigs allowed for complete submersion of the elastomeric chains in a water bath throughout the test period. The four groups of elastomeric chains were independently submerged in separate, $37^{\circ} \mathrm{C}$ water baths, housed in an incubator (ESPEC North America, Inc., Hudsonville, Michigan, United States) [Figure 2]. Four test measurements of remaining force were made at the following time intervals: initial (0), 1, 7, and 21 days [Figure 3]. Force measurements were obtained by Dontrix gauge 16oz (Ortho Organizer Inc., Carlsbad, California, United States) by a single, blinded examiner with the assistance of a second examiner.

\section{Statistical analysis}

Analysis of variance (ANOVA) was performed to analyze the dissimilarity between all groups in force decay. Data were analyzed by using the Statistical Package for the Social Sciences (SPSS) (IBM, Chicago, Illinois, United States).

\section{RESULTS}

The average and standard deviation of strength reduction in each group is presented in Table 1. A statistically

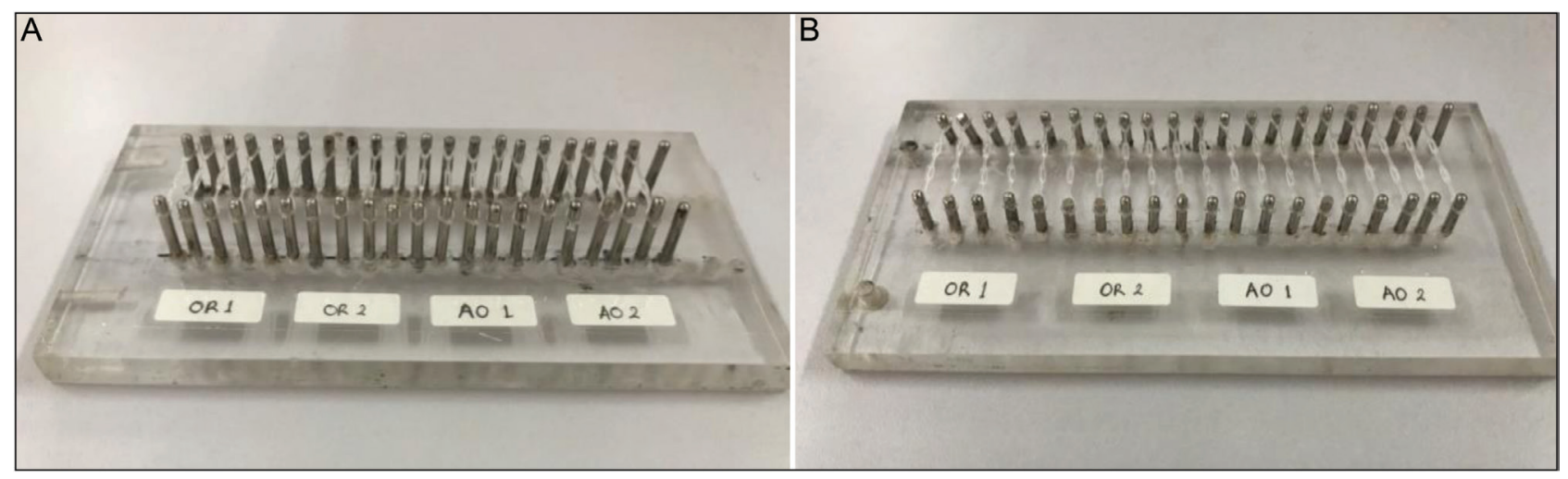

Figure 1: (A) Jig board with 20 sets of pins set $20 \mathrm{~mm}$ apart. (B) Elastomeric chains on jig board 
significant difference was observed between elastomeric chain groups in the strength reduction $(P<0.05)$ [Table 2]. The average initial strength obtained in each elastomeric chain was OR 1 of 376.5 , OR 2 of 373.78 , $\mathrm{AO} 1$ of 378.12 , and $\mathrm{AO} 2$ of $370.62 \mathrm{~g}$. From the results of research conducted, there was a decrease in the strength of elastomeric chains with significant thermoplastic and thermoset materials in the stretching time of $24 \mathrm{~h}, 7,14$, and 21 days. Strength reduction that occurs during the first 24 h of OR 1 decreased to 280.29 (25.4\%), OR 2 to $316.18(15.41 \%)$, AO 1 to $266.76(29.45 \%)$, and AO 2 to $301.10 \mathrm{~g}(18.76 \%)$.

The decrease in elastomeric chain strength on the seventh day showed that OR 1 decreased by 187.11 (50.3\%), OR2 by $283.5(24.15 \%)$, AO 1 by $157.44(58.36 \%)$, and $\mathrm{AO} 2$ by $209.79 \mathrm{~g}(43.4 \%)$. The decrease in elastomeric chain strength on day 14 showed that OR 1 decreased by 164.43

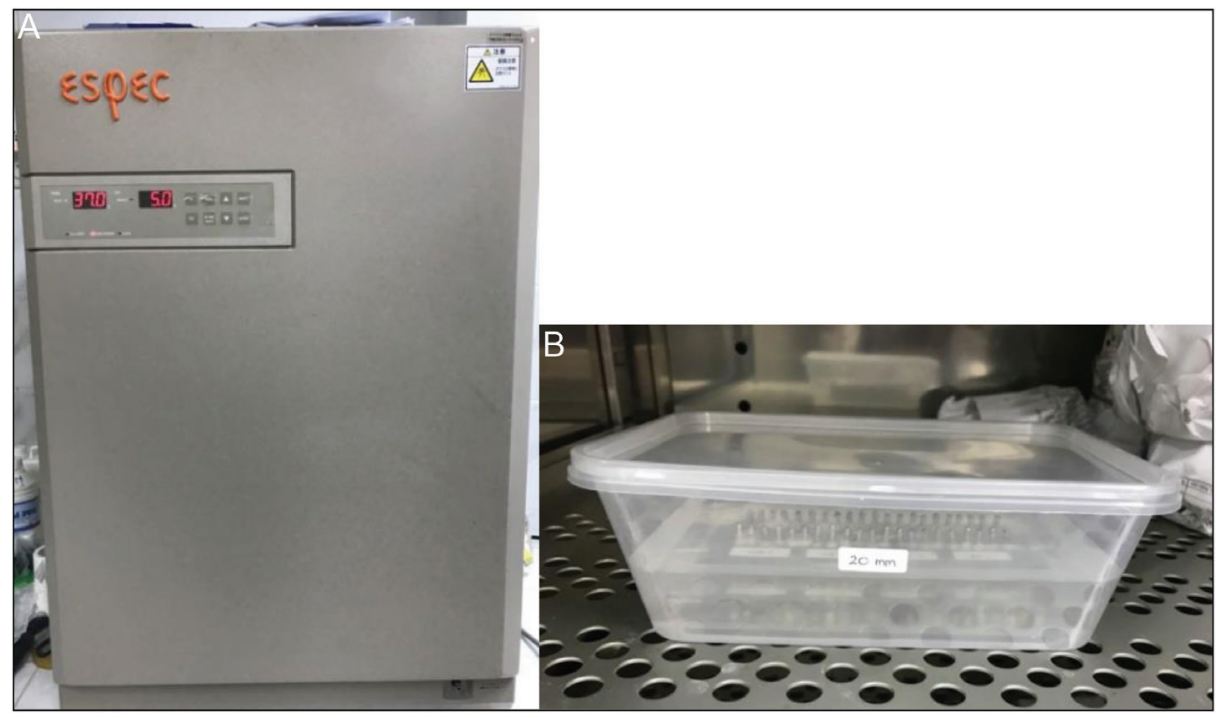

Figure 2: (A) Precision incubator with $37^{\circ} \mathrm{C}$ temperature (Espec, North America). (B) Elastomeric chains were submerged in water baths housed in an incubator

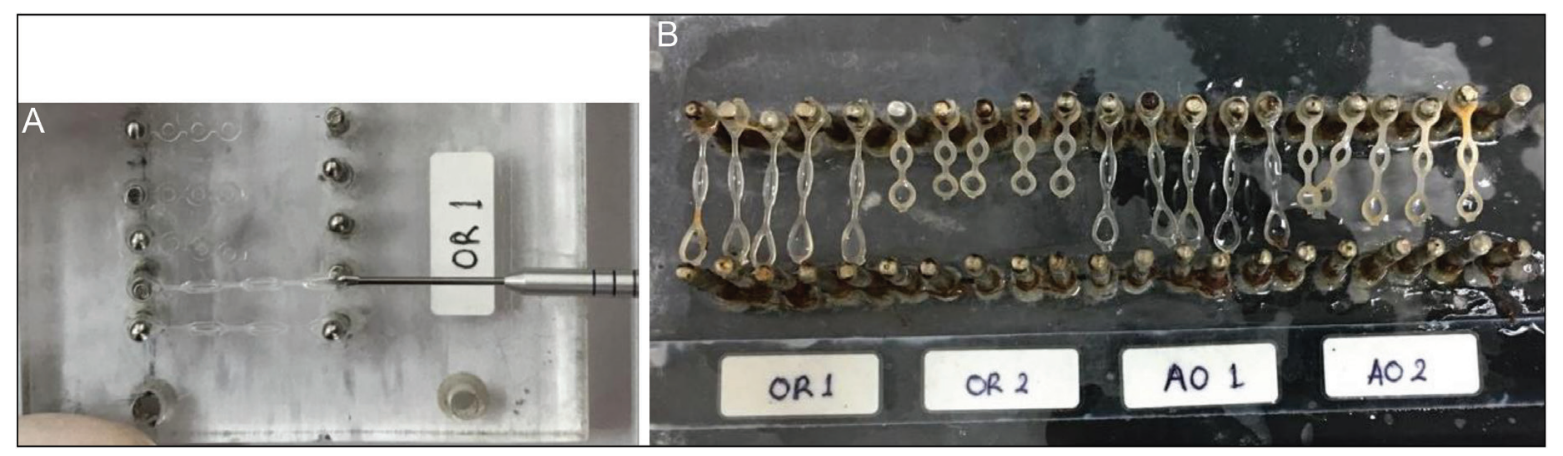

Figure 3: (A) Top view of elastomeric chains force levels being measured with Dontrix gauge. (B) Differences between four types of elastomeric chains in 21 days

\begin{tabular}{|c|c|c|c|c|c|c|}
\hline & $N$ & $\mathrm{Oh}$ & $24 \mathrm{~h}$ & 7 days & 14 days & 21 days \\
\hline \multirow[t]{2}{*}{ OR 1} & 5 & $376.5 \pm 3.94$ & $280.29 \pm 5.41$ & $187.11 \pm 15.52$ & $164.43 \pm 31.05$ & $147.42 \pm 23.7$ \\
\hline & & & $25,40 \%$ & $50,30 \%$ & $56,33 \%$ & $60,84 \%$ \\
\hline \multirow[t]{2}{*}{ OR 2} & 5 & $373.78 \pm 6.46$ & $316.18 \pm 6.71$ & $283.5 \pm 20.04$ & $249.48 \pm 12.67$ & $238.14 \pm 15.5$ \\
\hline & & & $15,41 \%$ & $24,15 \%$ & $33,25 \%$ & $36,28 \%$ \\
\hline \multirow[t]{2}{*}{ AO 1} & 5 & $378.12 \pm 5.94$ & $266.76 \pm 10.53$ & $157.44 \pm 14.33$ & $141.75 \pm 28.35$ & $130.41 \pm 15.5$ \\
\hline & & & $29,45 \%$ & $58,36 \%$ & $62,51 \%$ & $65,51 \%$ \\
\hline \multirow[t]{2}{*}{$\mathrm{AO} 2$} & 5 & $370.62 \pm 3.37$ & $301.1 \pm 6.87$ & $209.79 \pm 15.52$ & $164.43 \pm 23.7$ & $152.66 \pm 9.8$ \\
\hline & & & $18,76 \%$ & $43,40 \%$ & $55,63 \%$ & $58,80 \%$ \\
\hline
\end{tabular}

OR 1 = ORMCO thermoplastic, OR $2=$ ORMCO thermoset, AO 1 = American Orthodontics thermoplastic, AO $2=$ American Orthodontics thermoset 


\begin{tabular}{lcc}
\hline $\begin{array}{l}\text { Table 2: Statistically significant difference observed between } \\
\text { elastomeric chain groups in the strength reduction }\end{array}$ \\
\hline Immersion time & Elastomeric chains & $\begin{array}{c}\boldsymbol{P} \text { Value one-way ANOVA } \\
\text { test }\end{array}$ \\
\hline $24 \mathrm{~h}$ & OR 1, OR 2, AO 1, AO 2 & 0.000 \\
7 days & OR 1, OR 2, AO 1, AO 2 & 0.000 \\
14 days & OR 1, OR 2, AO 1, AO 2 & 0.000 \\
21 days & OR 1, OR 2, AO 1, AO 2 & 0.000 \\
\hline
\end{tabular}

ANOVA $=$ analysis of variance, OR $1=$ ORMCO thermoplastic, OR $2=$ ORMCO thermoset, $\mathrm{AO} 1=$ American Orthodontics thermoplastic, $\mathrm{AO} 2$ = American Orthodontics thermoset

There is significant difference between the decreases in the strength of the four elastomeric chains, both at the time of immersion $24 \mathrm{~h}, 7$ days, 14 days, and 21 days $(P<.05)$

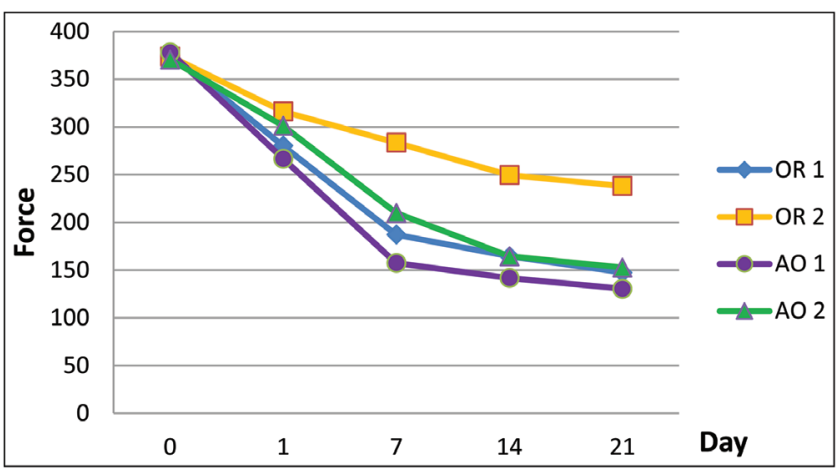

Figure 4: Comparing force decay between all groups of thermoplastic and thermoset elastomeric chains

(56.33\%), OR2 by $249.48(33.25 \%)$, AO 1 by 141.75 (62.51\%), and AO 2 by $164.43 \mathrm{~g}(56.33 \%)$. The decrease in elastomeric chain strength on day 21 showed that OR 1 experienced a decrease in strength of $147.42(60.84 \%)$, OR 2 of $238.14(36.28 \%)$, AO 1 of $130.41(65.51 \%)$, and AO 2 amounting to $152.66 \mathrm{~g}(58.8 \%)$ [Figure 4].

\section{Discussion}

Elastomeric chains are the most commonly used material in orthodontic treatment, especially for moving teeth. However, this material can experience a significant decrease in strength over time during the activation period. Several studies explain mechanical and environmental factors that can affect the decrease in the strength of various elastomeric chains. ${ }^{[8]}$

This study was carried out in vitro with experiments adjusted to the conditions in the oral cavity. Temperature and $\mathrm{pH}$ are adjusted according to the situation in the mouth, which is $37^{\circ} \mathrm{C}$ and saliva $\mathrm{pH} 6.7$ by immersing all the samples into a container and stored in an incubator. Each elastomeric chain is doubled in initial length stretching from $10 \mathrm{~mm}$ to $20 \mathrm{~mm}$ based on the average distance between the wing mesial cannula brackets and the premolar wing of the premolar brackets, as in the study of patterns of decreasing elastomeric chain strength. ${ }^{[4,9]}$
The results of research conducted are consistent with research conducted by Mirhashemi et al. where the decrease in strength after the first $24 \mathrm{~h}$ occurred at $20.7 \%-31.2 \%$ in elastomeric chains of thermoplastic materials and 9.3\%$12.4 \%$ in elastomeric chains of thermoset materials. ${ }^{[5,8]}$ The greatest decrease in strength that occurs after the first $24 \mathrm{~h}$ is also in accordance with the research conducted by Yagura et al. ${ }^{[10]}$ where the greater the initial strength given to elastomeric chains, the greater the decrease in strength that occurs. Buchmann et al. ${ }^{[3]}$ and Mirhashemi et al. ${ }^{[8]}$ suggested that the decrease in strength up to the seventh day was caused by absorption of saliva and formation, which coincided with hydrogen adhesion between water molecules and elastomeric macromolecules. ${ }^{[3,8]}$ The decrease in the strength of elastomeric chains is affected by the continuous absorption of fluids by elastomeric chains, which causes chain slippage and permanent changes in elastomeric chains. ${ }^{[1]}$

The significant different in strength degradation between elastomeric and thermoplastic chains due to the initial strength of thermoplastic material is greater than thermoset material to reach the $20 \mathrm{~mm}$ distance. According to research conducted by Weissheimer et al. ${ }^{[12]}$ the decrease in strength that occurs after the first $24 \mathrm{~h}$ is slowly and gradually until the third week where the decrease in strength is almost constant.

The strength range between 100 and $300 \mathrm{~g}$ is suggested by Ren et al. ${ }^{[13]}$ as the optimal strength for canine retraction. In this study, all thermoset elastomeric chains with initial strength range of 370.62-373.78 g have a residual strength range of 152.66-238.14 after immersion for 21 days, whereas the thermoplastic elastomeric chains with initial strength range of 376.5-378.12g have the remaining strength range of 130.41-147.42 $\mathrm{g}$ after immersion for 21 days.

We found that there was a higher strength reduction percentage after 21 days of thermoplastic compared to elastomeric material. Our result was in line with previous study that mentioned elastomeric thermoset material had a lower strength reduction compared to thermoplastic material. ${ }^{[14]}$

Several studies found that elastomeric chains with open or short modules produce greater elongation than closed or closed elastomeric chains, because greater lengthening is required for inter-modular links. ${ }^{[15,16]}$ Masoud et al. ${ }^{[14]}$ argue that thermoplastic materials are made of plastic and harden in greater temperatures. Meanwhile, thermoset materials become hard permanently during the process, cannot be destroyed, and have strong chemical bonds. This opinion supports research conducted where elastomeric chains of thermoplastic materials are stiffer and require initial stretching greater than elastomeric chains, which are more flexible thermoset materials and 
do not require greater stretching to achieve the desired distance.

The difference in the decline in strength in each elastomeric chain can be influenced by differences in the manufacturing process and the composition of elastomeric chains. Elastomeric chains with die-cut stamping and injection molding manufacturing processes have poly(ether) urethane composition and urethane poly(ester). ${ }^{[17]}$ Elastomeric chains produced with diecut stamping processes have more stable strength when compared to the injection molding process. Elastomeric chains of thermoset materials are examples of die-cut stamping. ${ }^{[6,14]}$

The composition contained in elastomeric chains according to Kardach et al. ${ }^{[16]}$ and Chenget al. ${ }^{[17]}$ with the largest constituent elements are $\mathrm{C}$ and $\mathrm{O}$. Element $\mathrm{C}$ adds strength and resistance to tear, abrasion, strain, and increases attractiveness, whereas elements of $\mathrm{O}, \mathrm{N}, \mathrm{Na}$, $\mathrm{Si}, \mathrm{Cl}$, and $\mathrm{Ca}$ are generally used as fillers, curing agents, amine-based accelerators, and additives. ${ }^{[18,19]}$

According to research conducted by Halimi et al. ${ }^{[20]}$ the decrease in the ideal strength of elastomeric chains is minimal with the remaining strength constant within the activation period. The greatest decrease in strength occurs in the first $24 \mathrm{~h}$, which then will occur a smaller decrease in strength every week and produce a decrease in strength of $50 \%-85 \%$ in 4 weeks. In this study, the mean strength decay at the third week is $40.63 \%$ for the elastomeric chains of thermoset material and $60.9 \%$ for the elastomeric chains of thermoplastic material.

Elastomeric chains with a more rigid thermoplastic material that requires greater strength in the initial withdrawal to achieve the desired distance. However, elastomeric chains of more flexible thermoset materials do not require early withdrawals that are too large to reach the desired distance so as to provide comfort to the patient. ${ }^{[21]}$

On the basis of the research conducted, the difference in strength of the four elastomeric chains is probably caused by the polymeric material contained in each elastomeric chain. The average strength remaining in the third week for elastomeric chains of thermoplastic materials is lower than for elastomeric chains of thermoset materials.

\section{Conclusion}

There was greater degradation of force in the first $24 \mathrm{~h}$ in all brand of elastomeric chain. The greater initial force of thermoplastic elastomeric chains will experience greater force decay compared to thermoset elastomeric chains. Thermoset elastomeric chains have greater residual force than thermoplastic elastomeric chains in 21 days stretching in artificial saliva solutions. The timing of orthodontic treatment control can be carried out for more than 21 days with the elastomeric chain thermoset material because these elastomeric chains still have the strength that can be used for canine retraction.

\section{Acknowledgement}

We thank Department of Orthodontics, Faculty of Dental Medicine, Universutas Airlangga, Surabaya, Indonesia for their support.

\section{Financial support and sponsorship \\ Nil.}

\section{Conflicts of interest}

There are no conflicts of interest.

\section{Ethical policy and institutional review board statement Not applicable.}

\section{Declaration of patient consent}

Not applicable.

\section{Data availability statement}

Data can be available on valid request on contacting to corresponding author mail.

\section{References}

1. Larrabee TM, Liu SS, Torres-Gorena A, Soto-Rojas A, Eckert GJ, Stewart KT. The effects of varying alcohol concentrations commonly found in mouth rinses on the force decay of elastomeric chain. Angle Orthod 2012;82:894-9.

2. Cuoghi OA, Tondelli PM, Mendonça MR, Aiello CA, Costa SC, Tanaka OM. Effect of different types of force on the amount of tooth movement, hyaline areas, and root resorption in rats. Eur J Gen Dent 2018;7:66-71.

3. Buchmann N, Senn C, Ball J, Brauchli L. Influence of initial strain on the force decay of currently available elastic chains over time. Angle Orthod 2012;82:529-35.

4. Quenzer JP, Lucato AS, Vedovello SAS, Valdrighi HC, Filho MV. Influence of elastic chain in the degradation of orthodontic forces: In vitro study. Rev Odontol UNESP 2015;44:320-25.

5. Nakhaei S, Agahi RH, Aminian A, Rezaeizadeh M. Discoloration and force degradation of orthodontic elastomeric ligatures. Dental Press J Orthod 2017;22:45-54.

6. Averous L, Bueno FC, HablotE, Garrigos MC, BocchiniS. Relationship between morphology, properties and degradation parameters of novative biobased thermoplastic polyurethanes obtained from dimer fatty acids. Polymer Degrad Stab 2012;97:1964-69.

7. Amin S, Amin M. Thermoplastic elastomeric (TPE) materials and their use in outdoor electrical insulation. Rev Adv Mater Sci 2011;29:25-30.

8. Mirhashemi AH, Farahmand N, Borujeni ES. Application of elastomeric chains in orthodontics: Past, present and future. Iranian J Orthod 2016; e5463:1-4.

9. Dittmer MP, Demling AP, Borchers L, Stiesch M, Kohorst P, Schwestka-Polly R. The influence of simulated aging on the mechanical properties of orthodontic elastomeric chains without an intermodular link. J Orofac Orthop 2012;73:289-97.

10. Yagura D, Baggio PE, Carreiro LS, Takahashi R. Deformation of elastomeric chains related to the amount and time of stretching. Dental Press J Orthod 2013;18:136-42.

11. Sulaiman TH, Eriwati YK, Indrani DJ. Effect of temperature on tensile force of orthodontics power chain in artificial saliva solution. J Phys Conf Ser 2018;1073:1-5. 
12. Weissheimer A, Locks A, de Menezes LM, Borgatto AF, Derech CD. In vitro evaluation of force degradation of elastomeric chains used in orthodontics. Dental Press J Orthod 2013;18:55-62.

13. Ren Y, Maltha JC, Kuijpers-Jagtman AM. Optimum force magnitude for orthodontic tooth movement: A systematic literature review. Angle Orthod 2003;73:86-92.

14. Masoud AI, Tsay TP, BeGole E, Bedran-Russo AK. Force decay evaluation of thermoplastic and thermoset elastomeric chains: A mechanical design comparison. Angle Orthod 2014;84:1026-33.

15. Dixit P, Jaipuria DK, Nagar, Jaipuria V. Invivo and invitro tensile properties of orthodontic elastomeric chains: A comparative study. Indian J Orthod Dentofac Res 2017;3:76-81.

16. Kardach H, Biedziak B, Olszewska A, Golusińska-Kardach E, Sokalski J. The mechanical strength of orthodontic elastomeric memory chains and plastic chains: An in vitro study. Adv Clin Exp Med 2017;26:373-8.
17. Cheng HC, Chen MS, Peng BY, Lin WT, Shen YK, Wang YH Surface Treatment on Physical Properties and Biocompatibility of Orthodontic Power Chains. BioMed Res Int 2017;2017:Article ID 6343724:1-9.

18. Martınez L, Alvarez L, Huttel Y, Mendez J, Roman E, Vanhulsel A, et al. Surface analysis of NBR and HNBR elastomers modified with different plasma treatments. Vacuum 2007:81:1489-92.

19. Martinez A, Lu Z, Handerson P, Picken SJ, Norder B, Imrie CT, Ripes A. Synthesis and characterisation of side chain liquid crystal copolymers containing sulfonic acid groups. Polymer 2012;53: 2604-12.

20. Halimi A, Benyahia H, Doukkali A, Azeroual MF, Zaoui F. A systematic review of force decay in orthodontic elastomeric power chains. Int Orthod 2012;10:223-40.

21. Long H, Wang Y, Jian F, Liao LN, Yang X, Lai WL. Current advances in orthodontic pain. Int J Oral Sci 2016;8:67-75. 